\title{
Tri-ortho-cresyl phosphate induces autophagy of mouse ovarian granulosa cells
}

\author{
Jinglei Wang ${ }^{1,2, *}$, Wenli Ruan ${ }^{3, *}$, Boshu Huang ${ }^{4}$, Shuxin Shao ${ }^{1}$, Dan Yang ${ }^{1}$, Mengling Liu ${ }^{1,5}$, \\ Lin Zeng ${ }^{1}$, Jie Wei ${ }^{1}$ and Jiaxiang Chen ${ }^{1,2}$ \\ ${ }^{1}$ Department of Physiology, Medical College of Nanchang University, Nanchang, People's Republic of China, \\ ${ }^{2}$ Jiangxi Provincial Key Laboratory of Reproductive Physiology and Pathology, Nanchang, People's Republic of \\ China, ${ }^{3}$ Department of Laboratory of Medicine, Guangzhou Zisheng Biotech, Guangzhou, People's Republic of \\ China, ${ }^{4}$ School of Public Health, Nanchang University, Nanchang, People's Republic of China and ${ }^{5}$ Nursing school \\ of Jiujiang University, Jiujiang, People's Republic of China
}

Correspondence should be addressed to J Chen; Email: chenjiaxiang@ncu.edu.cn

*(J Wang and W Ruan contributed equally to this work)

\begin{abstract}
Tri-ortho-cresyl phosphate (TOCP) has been widely used as plasticizers, plastic softeners and flame-retardants in industry and reported to have male reproductive toxicology. However, it is still unknown whether TOCP affects the female reproductive system and its underlying mechanism. In the present study, we found that TOCP exposure significantly decreased ovarian coefficient, caused disintegration and depletion of the granulosa cells in the ovary tissue and significantly inhibited the level of serum estradiol (E2). TOCP markedly increased both LC3-II and the ratio of LC3-II/LC3-I as well as autophagy proteins ATG5 and Beclin1 in the ovary tissue, implying that TOCP could induce autophagy in the ovary tissue. To further investigate the potential mechanism, primary ovarian granulosa cells were isolated in vitro and treated with $0-0.5 \mathrm{mM} \mathrm{TOCP}$ for $48 \mathrm{~h}$. We showed that TOCP decreased the number of viable mouse granulosa cells without affecting cell cycle and apoptosis of the cells. Intriguingly, TOCP treatment markedly increased both LC3-II and the ratio of LC3-II/LC3-I as well as ATG5 and Beclin1. Furthermore, transmission electron microscopy (TEM) showed that autophagic vesicles in the cytoplasm increased significantly in the TOCP-treated cells, indicating that TOCP could induce autophagy in the cells. Taken together, TOCP reduces the number of viable cells and induces autophagy in mouse ovarian granulosa cells without affecting cell cycle and apoptosis.

Reproduction (2019) 158 61-69
\end{abstract}

\section{Introduction}

Tri-ortho-cresyl phosphate (TOCP), one of the three isomers (i.e. $O-, m$ - or $p$-cresyl) of tricresyl phosphate (TCP), has been widely used as jet oil additives, plastic softeners and flame retardants in industry (Craig \& Barth 1999, Winder \& Balouet 2002). TOCP has been shown to induce a delayed neurodegenerative condition known as organophosphate-induced delayed neuropathy (OPIDN) by affecting the function of the central and the peripheral nerves in sensitive species such as humans and hens (Smith et al. 1930, Emerick et al. 2012).

TOCP has been reported to induce neurotoxicity (Craig \& Barth 1999, Zhang et al. 2007) and immunotoxicity (Brinkerhoff et al. 1981) in animals. In addition, many studies showed that TOCP can induce reproductive toxicology. TOCP has been shown to disrupt the seminiferous epithelium and inhibit spermatogenesis (Somkuti et al. 1987a, 1991, Chen et al. 2012). TOCP can also decrease the fertility index in Swiss (CD-1) mice and lead to a decrease in the number of liveborn pups per litter (Chapin et al. 1988). In our previous studies, we found that TOCP can decrease the number of viable rat spermatogonial stem cells (Liu et al. 2015) and saligenin cyclic-o-tolyl phosphate (SCOTP), a metabolite from TOCP by cytochrome P450, can also reduce the number of viable mouse spermatogonial stem cells (Chen et al. 2012). We also found TOCP can induce autophagy of rat spermatogonial stem cells (Liu et al. 2015). However, it is still unknown whether TOCP affects the female reproductive system and its underlying mechanism.

The main function of ovarian granulosa cells is to synthesize and secrete estrogen, as well as a myriad of growth factors, which play an important role in follicular growth, development and follicular atresia (Shimizu et al. 2009). Aromatase gene (CYP19) knockout interrupts the characteristic development of follicles in mice, indicating that estrogen is essential for normal ovarian function (Britt et al. 2000). Thereby, any alterations in the metabolism or function of ovarian 
granulosa cells may decrease the quality of the oocyte and the pregnancy chances (Hennet \& Combelles 2012). Numerous studies showed that many chemicals such as bisphenol A (BPA), perfluorooctanesulfonic acid (PFOS) and perfluorooctanoic acid (PFOA) can affect the function of ovarian granulosa cells. TOCP has been reported to decrease serum E2 concentration in female adult hen, which indicated that TOCP might affect the function of ovarian granulosa cells (Wu et al. 2000).

Macroautophagy, hereafter referred to as autophagy, is an evolutionary conserved lysosome-dependent catabolic pathway, which is responsible for the degradation of proteins and organelles during nutrient starvation (Kim et al. 2013). During the induction of autophagy, many autophagy proteins such as ATG5 and Beclin1 will be upregulated. Furthermore, cytosolic form of LC3 (LC3-I) will covalently conjugate with phosphatidylethanolamine (PE) and form LC3-II, the faster SDS-PAGE migrating form; LC3-II is then recruited and bound to autophagosome membrane (Kabeya et al. 2000, Liu et al. 2015). The conversion of LC3-I into LC3-II was regarded as a marker for the initiation of autophagy, while the amount of the LC3-II is related to the extent of autophagosome formation (Kabeya et al. 2000, Liu et al. 2015). In our previous studies, we found that TOCP could induce autophagy of human neuroblastoma SH-SY5Y cells (Chen et al. 2013), rat spermatogonial stem cells (Liu et al. 2015) and mouse Leydig TM3 cells (Liu et al. 2016). However, it is still unknown whether TOCP induces autophagy of ovarian granulosa cells.

The aim of the present study is to investigate the potential mechanism on TOCP-induced damage of mouse ovarian granulosa cells. This study sets in motion our future investigation of the underlying mechanism on TOCP-induced female reproductive toxicology.

\section{Materials and methods}

\section{Reagents}

TOCP was purchased from BDH Chemicals Co. Ltd (Poole, England). Cell culture reagents were obtained from Gibco BRL. TRIzol reagent was purchased from Invitrogen Life Technologies and EasyScript ${ }^{\mathrm{TM}}$ First-strand cDNA Synthesis SuperMix for RT-PCR reaction was purchased from TransGen Biotechnology (Beijing, China). Annexin V-FITC Apoptosis Detection Kit was obtained from Invitrogen Life Technologies. Rabbit anti-follicle-stimulating hormone receptor (FSHR) polyclonal antibody (ab150557) was purchased from Abcam. Rabbit anti-LC3 polyclonal antibody (PD014), rabbit antiBeclin 1 polyclonal antibody (PD017) and rabbit anti-ATG5 polyclonal antibody (PM050) were obtained from MBL Co. Ltd (Nagoya, Japan). Mouse anti- $\beta$-actin monoclonal antibody was purchased from Santa Cruz Biotechnology. The enhanced chemiluminescence $(\mathrm{ECL})$ reagent was obtained from Pierce Biotechnology.

\section{Animal administration}

Adult female Kunming (KM) mice (8 weeks old, 20-25g) were orally administrated at different concentrations of TOCP $(0,100,200,400 \mathrm{mg} / \mathrm{kg} /$ day, dissolved in corn oil) for 14 days, blood samples were collected by cardiocentesis and the serum was then separated and stored at $-20^{\circ} \mathrm{C}$. Then, the ovaries and uterus were quickly dissected and weighed; the ovary was fixed in formalin solution for hematoxylin and eosin staining or frozen in liquid nitrogen before storing at $-80^{\circ} \mathrm{C}$ for western blotting analysis. All animal procedures were performed in compliance with China's current legislation and approved by the Animal Ethics Committee of Nanchang University.

\section{HE staining and follicles counting}

Female mice ovaries were dissected, fixed in formalin solution, embedded in paraffin, sectioned $(4 \mu \mathrm{m})$ and stained with hematoxylin and eosin according to the method described by Matzuk et al. (1992). Twelve ovaries (six mice per group) from each group were utilized to count the follicles; then five consecutive sections were made from each ovary. The mean value of the 60 sections was considered as the number of follicles per section in each treatment group. This analysis was done blind to the treatment groups. Follicles were classified as follows: primordial follicle (POF, oocyte surrounded by one layer of flattened pre-granulosa cells), primary follicle (PF, oocyte surrounded by a single layer of cuboidal granulosa cells), secondary follicle (SF, oocyte surrounded by more than one layer of cuboidal granulosa cells) and antral follicle (AF, more than four layers of granulosa cells with one or more clearly defined antral spaces or with a cumulus granulosa cell layer). In some cases, AFs showed no antral space in cross-section analysis but were considered antral if they contained $\geq 5$ granulosa cell layers (Yang et al. 2007). The follicular granulosa cells were intact and ordered in the normal follicles, while the arrangement of the granulosa cells was disordered in the abnormal follicles.

\section{Detection of estradiol content by ELISA}

The blood samples were centrifuged at $12,000 \mathrm{~g}$ at $4{ }^{\circ} \mathrm{C}$ for $30 \mathrm{~min}$ to separate serum. The concentration of estradiol (E2) in the serum was determined using an ELISA kit according to the manufacturer's instructions (Shanghai Bangyi Biotechnology Co Ltd, Shanghai, China).

\section{Isolation and culture of primary ovarian granulosa cells}

Primary ovarian granulosa cells were isolated from 14-day-old female mice according to the method described by Canipari et al. (1987). Briefly, ovarian granulosa cells were isolated by needle puncture under the microscope specifically from preantral and small AFs (puncturing of large AFs was avoided). The cells were then maintained in Dulbecco's modified Eagle's medium/F-12 medium supplemented with $10 \%$ fetal bovine serum, $100 \mathrm{IU} / \mathrm{mL}$ penicillin and $100 \mu \mathrm{g} / \mathrm{mL}$ streptomycin under $37^{\circ} \mathrm{C}, 5 \% \mathrm{CO}_{2}$ condition. 


\section{Immunocytofluorescence staining}

Primary ovarian granulosa cells were harvested on glass coverslips, fixed in $4 \%$ paraformaldehyde, blocked in 3\% BSA for $1 \mathrm{~h}$ at room temperature, and then incubated with rabbit anti-FSHR polyclonal antibody (1:100 dilution) overnight at $4^{\circ} \mathrm{C}$. The cells were washed three times with PBS for $5 \mathrm{~min}$ and then incubated with rhodamine/TRITC-conjugated secondary antibodies (1:200 dilution). Nuclei was stained with 4, 6-diamidoino-2-phenylindole (DAPI). The images were captured with a fluorescence microscope (Nikon TE-2000).

\section{Cell counts}

The cells $\left(1 \times 10^{4}\right.$ cells/well $)$ were seeded in a 96-well culture plate and were incubated with fresh medium containing 0-0.5 mM TOCP for $48 \mathrm{~h}$. TOCP were dissolved in DMSO, and the final concentration of DMSO in the culture medium was $0.1 \%(\mathrm{v} / \mathrm{v})$. Forty-eight hours later, cell counts were assessed by using a hemocytometer.

\section{MTT reduction assay}

The cells $\left(1 \times 10^{4}\right.$ cells/well $)$ were seeded in a 96-well culture plate and were incubated with fresh medium containing 0-0.5 mM TOCP for $48 \mathrm{~h}$. Cell viability was then assessed by the MTT assay. Briefly, cell medium containing $0.5 \mathrm{mg} / \mathrm{mL}$ MTT was replaced to each well and incubated at $34^{\circ} \mathrm{C}$ in $5 \%$ $\mathrm{CO}_{2} / 95 \%$ air for $4 \mathrm{~h}$; MTT, a yellow tetrazole, was then reduced to purple insoluble formazan in living cells via mitochondrial dehydrogenase activity. The formed formazan would become a colored solution when it was dissolved in DMSO, and the absorbance was measured in a spectrophotometer at $490 \mathrm{~nm}$.

\section{Flow cytometric analysis}

The cells were seeded at a density of $1 \times 10^{5}$ in $60 \mathrm{~mm}$ culture dishes. After $24 \mathrm{~h}$, cells were treated with $0-0.5 \mathrm{mM}$ TOCP for $48 \mathrm{~h}$ in complete medium. At the indicated time, cells were harvested by trypsinization, centrifuged at $600 \mathrm{~g}$ for $5 \mathrm{~min}$, washed in cold PBS, and fixed overnight in $75 \%$ cold ethanol, digested with $20 \mu \mathrm{g} / \mathrm{mL}$ RNase A and stained with $50 \mu \mathrm{g} / \mathrm{mL}$ PI. The cells were then subjected to flow cytometric analysis on a FACScan (Becton Dickinson Company).

\section{Reverse transcription-polymerase chain reaction}

The cells were plated at a density of $5 \times 10^{5}$ in $60 \mathrm{~mm}$ culture dishes and were incubated with fresh medium containing 0-0.5 mM TOCP for $48 \mathrm{~h}$. Total RNA was isolated using TRIzol reagent according to the manufacturer's instructions and quantified spectrophotometrically. The polymerase chain reaction (PCR) analyses were then performed according to the method described by Liu et al. (2015).

\section{Hoechst staining}

Coverslips with mouse ovarian granulosa cells were collected and fixed with $4 \%$ paraformaldehyde and stained with Hoechst $33342(5 \mu \mathrm{g} / \mathrm{mL})$ for $20 \mathrm{~min}$ at room temperature.
The coverslips were washed, mounted on glass slides and observed by fluorescence microscopy.

\section{Annexin V-FITC/PI apoptosis assay}

Apoptosis was observed by flow cytometry using an AnnexinVFITC Apoptosis Kit as described previously (Liu et al. 2016).

\section{Western blotting analysis}

The decapsulated and homogenized ovary tissue and the mouse ovarian granulosa cells were harvested in ice-cold RIPA lysis buffer containing protease inhibitor cocktail. Equal amounts of cell lysate were utilized for western blotting analysis as described previously (Liu et al. 2016).

\section{Transmissiovn electron microscopy analyses}

The cells were incubated in normal DMEM medium or in starvation medium (Hank's buffered salt solution (HBSS) with $20 \mathrm{mM}$ HEPES, $\mathrm{pH} 7.15$ ) for $3 \mathrm{~h}$ or treated with $0.5 \mathrm{mM}$ TOCP for $48 \mathrm{~h}$; the cell monolayers were washed with PBS and scraped gently with a plastic cell scraper. Then, the harvested cells were pelleted by centrifugation at $800 \mathrm{rpm}$ for $10 \mathrm{~min}$ and fixed in ice-cold 2.5\% glutaraldehyde for $2 \mathrm{~h}$. Afterward, samples were postfixed in $1 \% \mathrm{OsO} 4$ for $1 \mathrm{~h}$, dehydrated through ethanol series and embedded in epoxy resin, and then ultra-thin sections $(60 \mathrm{~nm})$ were double stained with uranyl acetate and lead citrate. Representative areas were examined by transmission electron microscope (Hitachi H800).

\section{Statistical analysis}

Values are expressed as means \pm S.E. Data were evaluated by one-way ANOVA with a Newman-Keuls multiple range test. For each test, $P$ values less than $0.05(P<0.05)$ were considered statistically significant.
A

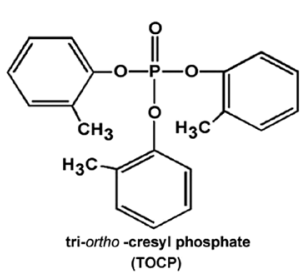

C

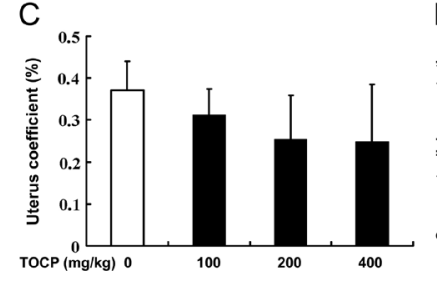

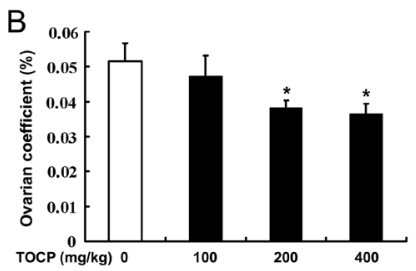

D

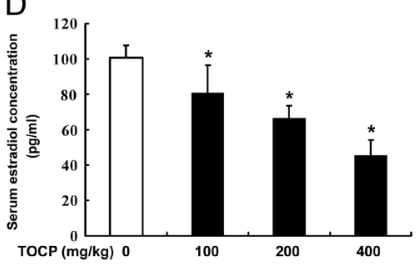

Figure 1 Effect of TOCP on the mice ovarian and uterus coefficient and estradiol concentration. (A) Chemical structure of tri-orthocresylphosphate (TOCP). Female mice were treated with 0, 100, 200 or $400 \mathrm{mg}$ TOCP/kg/day for 14 days. The ovarian (B) and uterus (C) coefficient is the ovary and uterus weight expressed as a percentage of body weight, respectively. (D) The serum estradiol concentration was detected by ELISA. Data were analyzed by one-way ANOVA. $n=6$ mice, ${ }^{*} P<0.05$. 

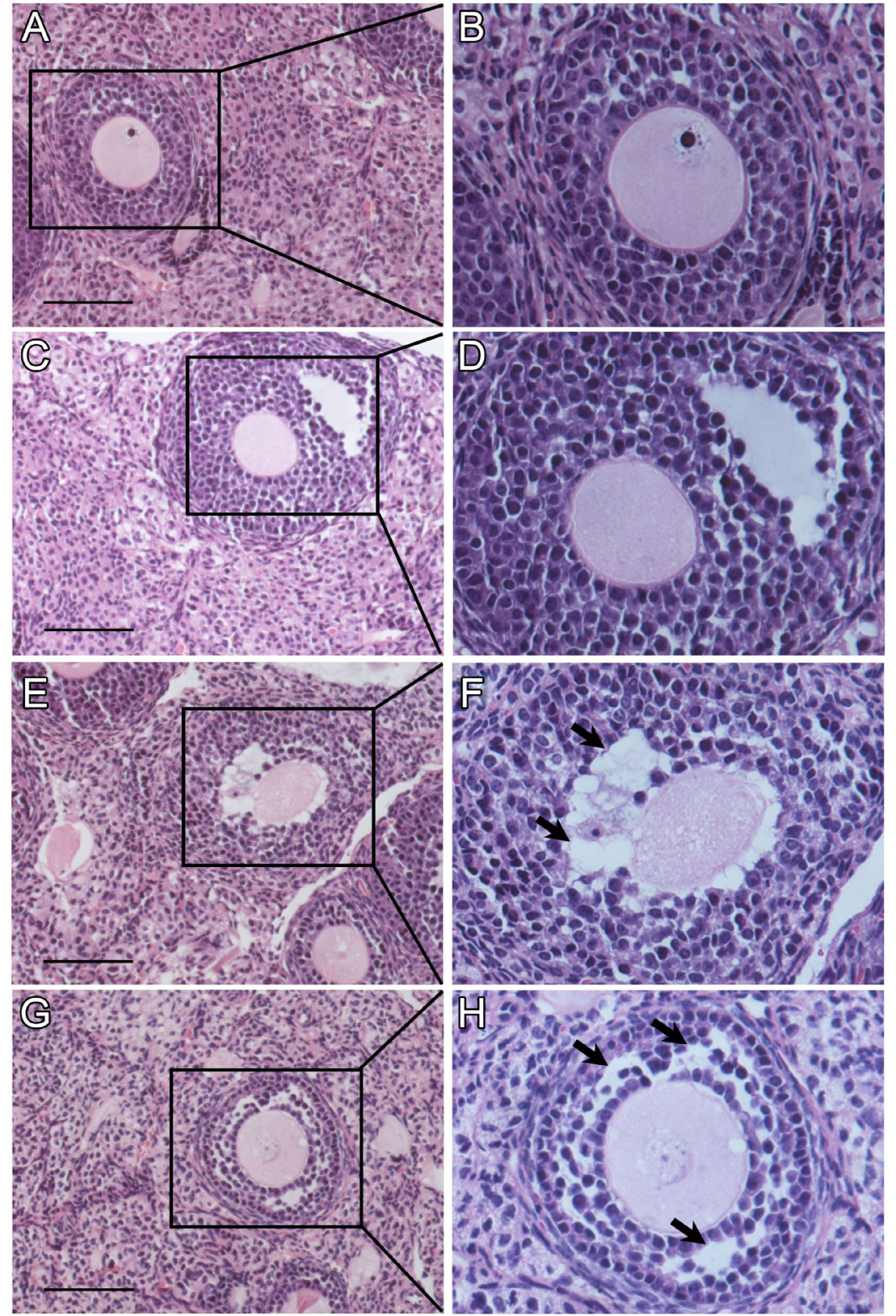
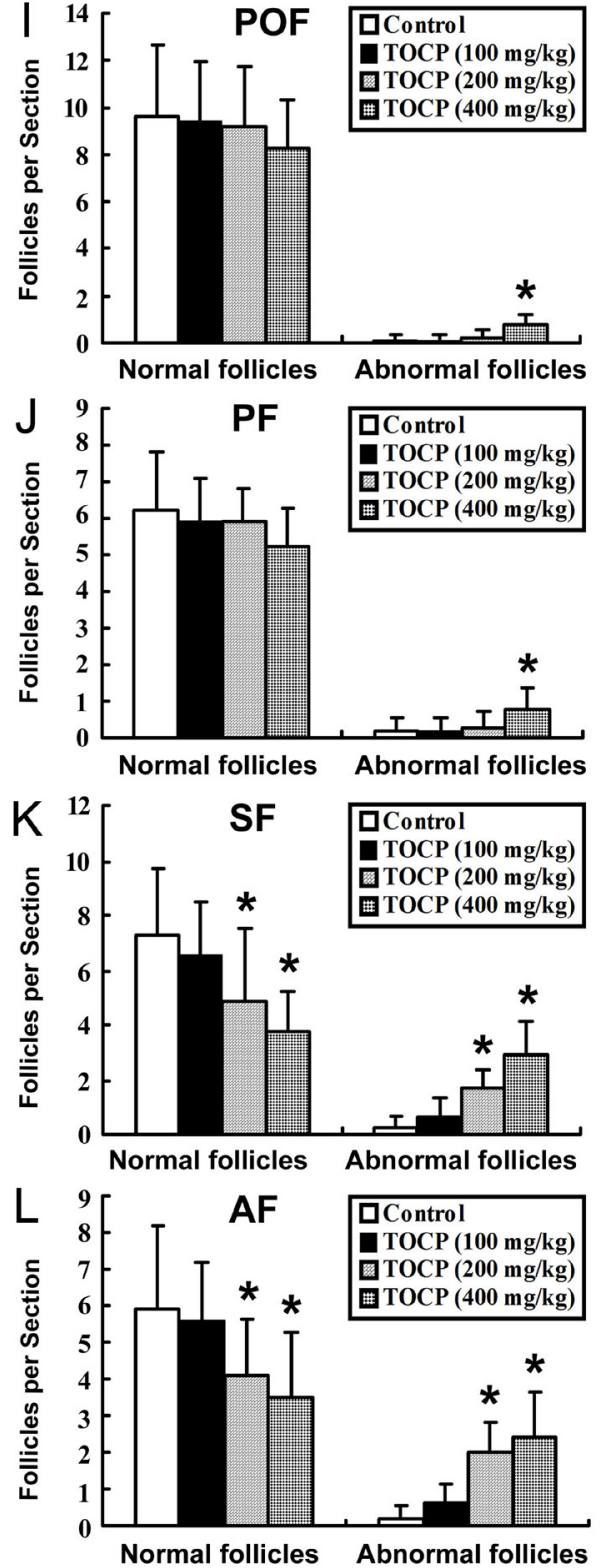

Figure 2 Effect of TOCP exposure on mouse ovarian histopathology. Ovaries were obtained from female mice treated with 0 (A and B), 100 ( $\mathrm{C}$ and $\mathrm{D}), 200$ ( $\mathrm{E}$ and $\mathrm{F}$ ) or $400(\mathrm{G}$ and $\mathrm{H}) \mathrm{mg}$ TOCP/kg/day for 14 days; the ovaries were stained with hematoxylin and eosin and then were visualized under a IX51 Olympus microscope. Magnification: 100x. Size bars indicate $100 \mu \mathrm{m}$. The follicular granulosa cells were intact and ordered in the normal follicles, while the arrangement of the granulosa cells was disordered in the abnormal follicles. The black arrows indicate the abnormal follicle. Normal and abnormal follicles at each stage of follicle including (I) primordial follicles (POF), (J) primary follicles (PF), (K) secondary follicles (SF) and (L) antral follicles (AF) were counted. Data were analyzed by one-way ANOVA. $n=6$ mice, ${ }^{*} P<0.05$.

\section{Results}

\section{Effect of TOCP exposure on ovarian histopathology in mice}

In order to investigate the effect of TOCP on ovarian histopathology, female mice were administrated with the indicated concentrations of $\operatorname{TOCP}(0,100,200$, $400 \mathrm{mg} / \mathrm{kg} /$ day) for 14 days (Chen et al. 2012). As shown in Fig. 1, TOCP exposure significantly decreased ovarian coefficient. Meanwhile, estradiol concentration in the serum was also decreased in the TOCP-treated group. These results implied that TOCP could cause ovarian granulosa cells damage. To further confirm the underlying effect, the ovaries were observed by hematoxylin and eosin (HE) staining. As shown in Fig. 2, the arrangement of follicular granulosa cells was disordered in the 200 and $400 \mathrm{mg} / \mathrm{kg} /$ day TOCP exposure group. The number of abnormal follicles at each stage of follicle including POF, PF, SF and AF increased in the 200 and $/$ or $400 \mathrm{mg} / \mathrm{kg} /$ day TOCP 
A

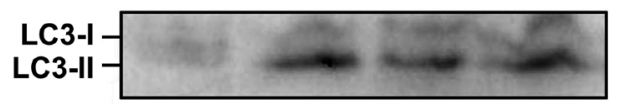

ATG5

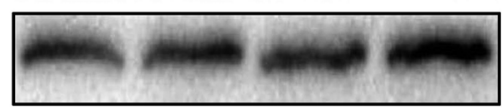

Beclin1

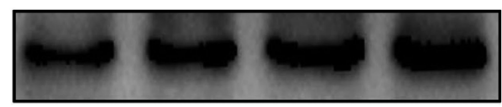

\section{Actin}

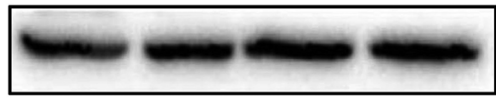

$\operatorname{TOCP}(\mathrm{mg} / \mathrm{kg}) \quad 0$

100

200

400

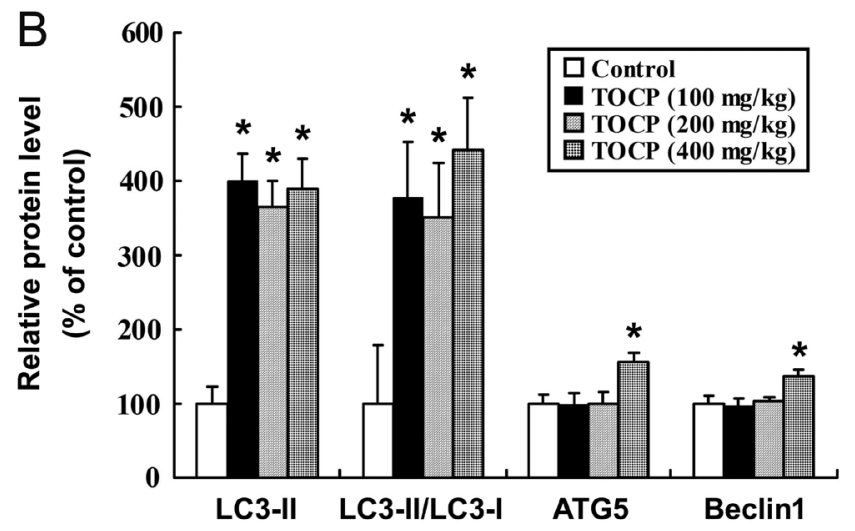

Figure 3 TOCP exposure induces autophagy of ovarian tissue. (A) Ovaries were obtained from female mice treated with $0,100,200$ or $400 \mathrm{mg}$ TOCP/kg/day for 14 days. (A) The protein levels of LC3, ATG5 and Beclin1 were observed by western blot; Actin was used as an internal control. (B) The relative protein levels were quantified by densitometry. Data were analyzed by one-way ANOVA. $n=6$ mice, ${ }^{*} P<0.05$.

exposure group. To further detect the potential ovarian damage mechanisms of TOCP, we evaluated the autophagy levels in mouse ovarian tissues. As shown in Fig. 3, there was a significant increase in the autophagy proteins such as LC3-II, Beclin1 and ATG5 in the TOCP-treated group, indicating that TOCP could induce autophagy in the testis tissue. These results indicated that autophagy might be involved in TOCP-induced atrophy of granulosa cells in the ovary.

\section{TOCP decreases the number of viable mouse ovarian granulosa cells}

To confirm whether TOCP can cause damage of ovarian granulosa cells, primary ovarian granulosa cells were isolated and identified with FSHR fluorescence staining. The purity of the isolated ovarian granulosa cells is about 90\% (Fig. 4A). We treated mouse ovarian granulosa cells with $0-0.5 \mathrm{mM}$ TOCP for $48 \mathrm{~h}$. As shown in Fig. 4B and $\mathrm{C}$, TOCP reduced the number of viable mouse ovarian granulosa cells in a dose-dependent manner.

\section{TOCP induces autophagy of mouse ovarian granulosa cells}

To evaluate whether TOCP induces autophagy of mouse ovarian granulosa cells, the cells were treated with indicated concentration of TOCP for $48 \mathrm{~h}$, the autophagy proteins LC3, ATG5 and Beclin 1 were analyzed by western blot. As shown in Fig. 5A and B, treatment with TOCP could dramatically increase the protein level of LC3-II and the ratio of LC3-II/ LC3-I, as well as ATG5 and Beclin 1, implying that TOCP could induce autophagy of mouse ovarian granulosa cells. Autophagy was further observed by transmission electron microscopy (TEM), which is the 'gold' standard method. There were relatively few autophagosomes in the cytoplasm of the control cells, while the autophagic vesicles containing extensively degraded organelles such as mitochondria and endoplasmic reticulum increased significantly in the TOCP or starvation-treated group (Fig. 5C and D). These results indicated that TOCP induced autophagy of mouse ovarian granulosa cells.
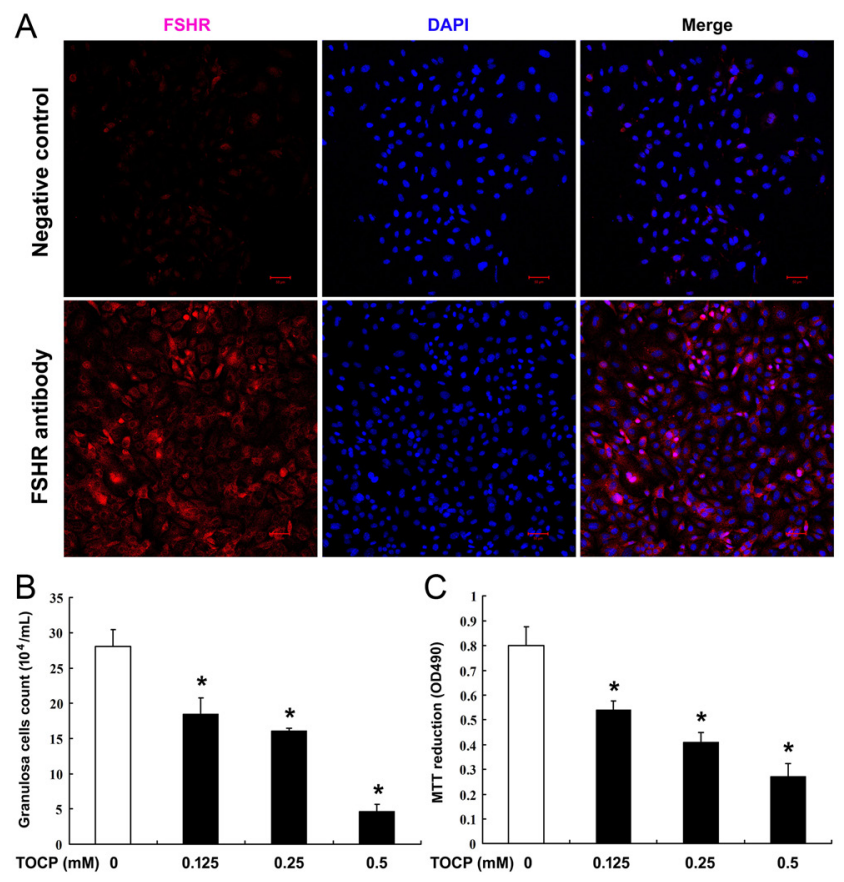

Figure 4 TOCP decreases the number of viable mouse ovarian granulosa cells. (A) The isolated primary ovarian granulosa cells were identified with rabbit anti-follicle stimulating hormone receptor (FSHR) polyclonal antibody. Mouse ovarian granulosa cells were treated with $0-0.5 \mathrm{mM}$ TOCP for $48 \mathrm{~h}$. Then, cell counts (B) and cell viability (C) were assessed by using a hemocytometer and MTT (3-(4,5-dimethylthiazol-2-yl)-2,5-diphenyltetrazolium bromide) assay, respectively. The experiment was done in triplicate and repeated three times. Data were analyzed by one-way ANOVA. $* P<0.05$. 
A

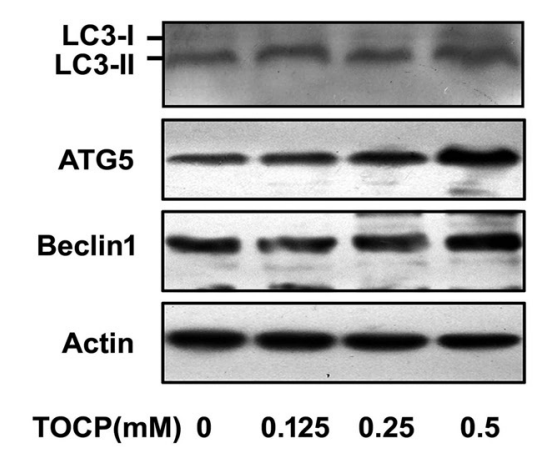

C

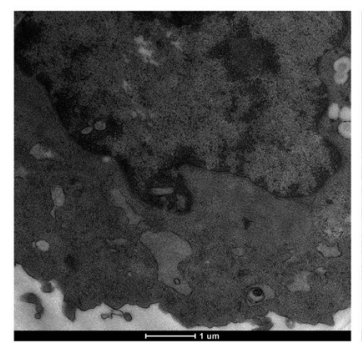

Control

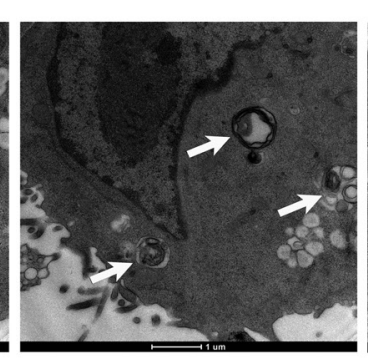

TOCP (0.5 mM)

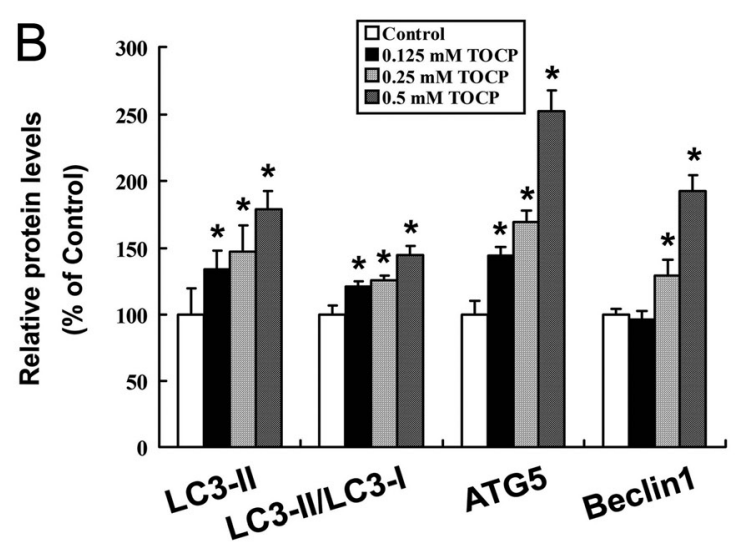

D
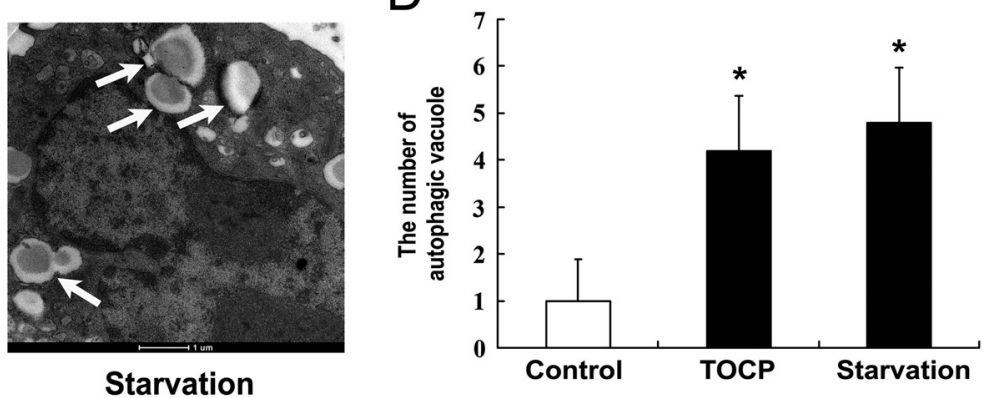

Figure 5 TOCP induces autophagy of mouse ovarian granulosa cells. (A) Mouse ovarian granulosa cells were treated with 0-0.5 mM TOCP for $48 \mathrm{~h}$; the protein levels of LC3, ATG5 and Beclin1 were observed by western blot. (B) The relative protein levels were quantified by densitometry and expressed as percentage of the control cells. (C) The cells were incubated in normal DMEM medium or in starvation medium (Hank's buffered salt solution (HBSS) with $20 \mathrm{mM}$ HEPES, $\mathrm{pH}$ 7.15) for $3 \mathrm{~h}$ or treated with $0.5 \mathrm{mM}$ TOCP for $48 \mathrm{~h}$; autophagic vesicles in the cells were visualized by transmission electron microscopy (TEM), the autophagic vacuoles are indicated by arrows. (D) Quantitative results showing accumulated autophagic vacuole ultrastructures in the cells. The experiment was done in triplicate and repeated three times. Data were analyzed by one-way ANOVA. ${ }^{*} P<0.05$.

\section{Effect of TOCP on cell cycle of mouse ovarian granulosa cells}

To determine whether TOCP affects cell cycle of mouse ovarian granulosa cells, the G1, S and G2/M phases were determined by flow cytometry after the cells were treated with $0-0.5 \mathrm{mM}$ TOCP for $48 \mathrm{~h}$. Compared with control group, TOCP did not affect the cell cycle significantly (Fig. 6A). The mRNA levels of certain cyclins and cyclindependent kinase inhibitor (CKIs) family members such as p21, p27, p53 and cyclin D1, which were crucially required for the regulation of cell cycle progression, were further observed by RT-PCR. As shown in Fig. 6B and $C$, there was no significant change in the mRNA levels of p21, p27, p53 and cyclin D1 between the control and TOCP-treated cells.

\section{Effect of TOCP on apoptosis of mouse ovarian granulosa cells}

As shown in Fig. 6A, we did not find the apoptotic cell death marker sub-G1 peak from cell cycle distribution in all of the tested cells, suggesting that the anti-proliferative effect of TOCP did not result from the induction of apoptotic cell death. In order to further confirm the hypothesis, apoptosis was detected by Hoechst staining and annexin V/PI double staining, respectively. As shown in Fig. 7A, B and C, TOCP did not induce apoptosis of mouse ovarian granulosa cells.

\section{Discussion}

In the current study, we provided evidence for the first time that TOCP decreased the number of viable mouse ovarian granulosa cells and induced autophagy with no effect on cell cycle progression and apoptosis.

As one of the three tricresyl phosphate (TCP) isomers, TOCP, has been shown to cause male reproductive toxicology. TOCP can inhibit sperm motility and decrease sperm number in roosters (Somkuti et al. 1987b) and rats (Somkuti et al. 1991, Latendresse et al. 1994). In our previous study, we found that TOCP could disrupt the seminiferous epithelium in the mouse testis and decrease the sperm density in the epididymis (Chen et al. 2012). TOCP can also decrease the fertility index in Swiss (CD-1) mice (Chapin et al. 1988). TOCP has been 

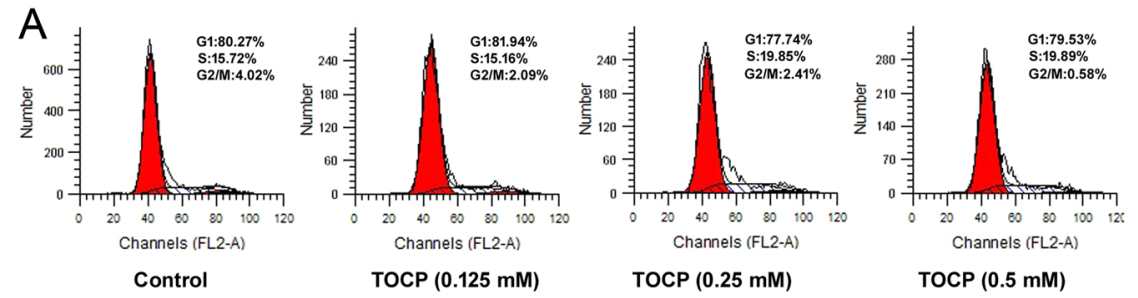

TOCP (0.5 mM)
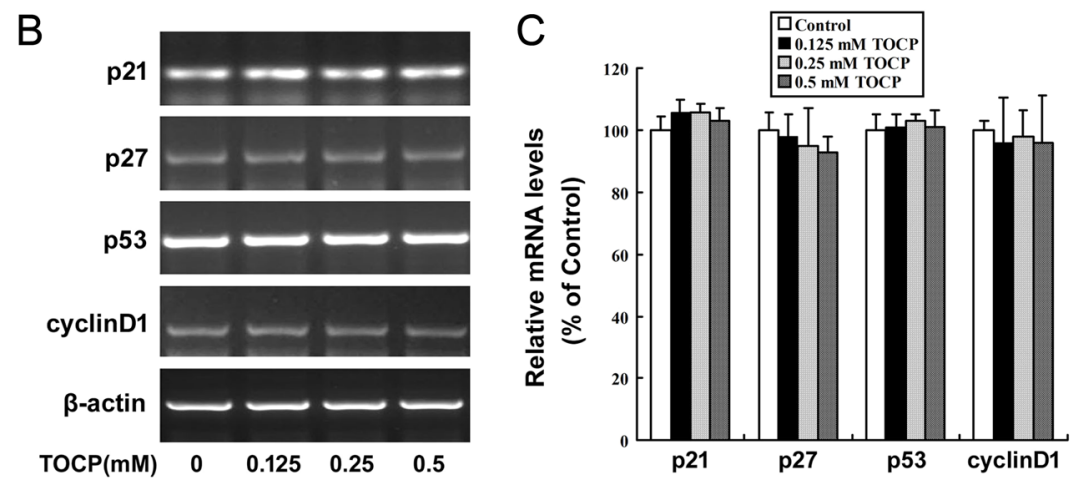

Figure 6 Effect of TOCP on the cell cycle of mouse ovarian granulosa cells. (A) Mouse ovarian granulosa cells were treated with 0-0.5 mM TOCP for $48 \mathrm{~h}$; then cell cycle was observed by FCM assay. (B) The mRNA levels of p21, p27, p53 and cyclin D1 were observed by RT-PCR; the actin was used as an internal control. (C) The relative mRNA levels were quantified by densitometry and expressed as percentage of the control cells. The experiment was done in triplicate and repeated three times. Data were analyzed by one-way ANOVA.

reported to decrease serum E2 concentration in female adult hen, which indicated that TOCP might affect female reproductive system (Wu et al. 2000). However, the underlying mechanism is still unknown. In this study, TOCP exposure significantly decreased ovarian coefficient. Hematoxylin and eosin (HE) staining showed that TOCP exposure could disorder the arrangement of follicular granulosa cells and increase the number of abnormal follicles at each stage of follicle including POF, $\mathrm{PF}$, SF and AF, which indicated that TOCP could lead to atrophy of granulosa cells in the ovary. One important function of ovarian granulosa cells is to synthesize and secrete estradiol, so we detected the estradiol concentration in the serum. In the present study, we found that TOCP decreased significantly serum estradiol concentration, which indicated that TOCP could cause ovarian granulosa cell damage. In our previous studies, we found that TOCP could inhibit the viability of many kinds of cells such as rat spermatogonial stem cells (Liu et al. 2015), mouse Leydig TM3 cells (Liu et al. 2016) and mouse liver cancer Hepa 1-6 cells (Xu et al. 2016). In the current study, we found that the number of viable primary mouse ovarian granulosa cells was reduced by TOCP in a dose-dependent manner.

Autophagy is a lysosome-dependent catabolic pathway, which is responsible for the degradation of proteins and organelles to protect the cells against the accumulation of damaged organelles or protein aggregates; however, autophagy can also lead to cell death (Scarlatti et al. 2009, Liu et al. 2015). Many chemicals have been shown to induce autophagy (Yao et al. 2016, Quan et al. 2017, Mahemuti et al. 2018, Sun et al. 2018). TOCP was found to induce autophagy of human neuroblastoma SH-SY5Y cells (Chen et al. 2013), rat spermatogonial stem cells (Liu et al. 2015) and mouse Leydig TM3 cells (Liu et al. 2016). However, there is no report on whether TOCP induces autophagy
A
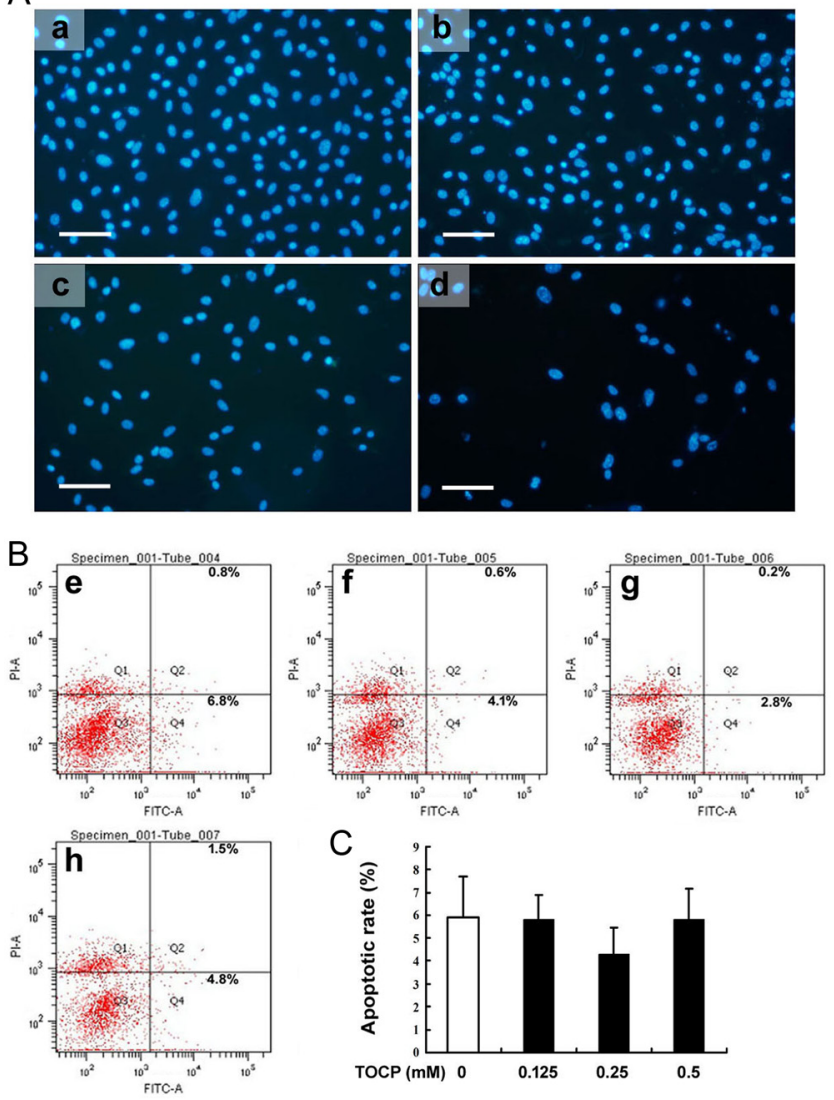

Figure 7 Effect of TOCP on apoptosis of mouse ovarian granulosa cells. Mouse ovarian granulosa cells were treated with $0 \mathrm{mM}$ (a and e), $0.125 \mathrm{mM}$ (b and f), $0.25 \mathrm{mM}$ (c and g) or $0.5 \mathrm{mM}$ ( $\mathrm{d}$ and $\mathrm{h}$ ) TOCP for $48 \mathrm{~h}$. Then, cell apoptosis was observed by Hoechst staining (A) and FCM assay (B and C), respectively. The experiment was done in triplicate and repeated three times. Data were analyzed by one-way ANOVA. Size bars in panel A indicate $20 \mu \mathrm{m}$. 
of mouse ovarian granulosa cells. In the present study, we detected autophagy proteins LC3, ATG5 and Beclin1 in mouse ovarian tissues by western blot. We found that TOCP exposure significantly increased the protein levels of LC3-II, Beclin1 and ATG5, indicating that TOCP could induce autophagy in the testis tissue. These results indicated that autophagy might be involved in TOCP-induced atrophy of granulosa cells in the ovary. To further determine whether TOCP induces autophagy of mouse ovarian granulosa cells, primary ovarian granulosa cells were utilized for subsequent detection of autophagy. We found that TOCP exposure could significantly increase the protein level of LC3-II and the ratio of LC3-II/LC3-I, as well as ATG5 and Beclin1. Furthermore, the autophagic vesicles increased significantly in the TOCP-treated group. These results indicated that TOCP induced autophagy of mouse ovarian granulosa cells.

The inhibition effect of TOCP might also result from the cell cycle arrest (Long et al. 2008). To confirm this hypothesis, mouse ovarian granulosa cells were observed by flow cytometry and RT-PCR. We found that the indicated concentration of TOCP had no significant effect on the population of cells in G1 phase, $S$ phase or $G_{2} / M$ phase and the mRNA levels of cell cycle related proteins, such as cyclin D1, p21, p27 or p53. Hoechst staining and annexin V-FITC/PI dual staining showed that TOCP did not induce apoptosis of the cells. Taken together, these results indicated that TOCP had no effect on cell cycle and apoptosis of mouse ovarian granulosa cells, which is consistent with our previous study in rat spermatogonial stem cells (Liu et al. 2015).

In summary, we have shown that TOCP decreases the number of viable mouse ovarian granulosa cells and induces autophagy with no effect on cell cycle and apoptosis. This study sets in motion our future investigation of the underlying molecular mechanisms on TOCP-induced toxicity of female reproductive system.

\section{Declaration of interest}

The authors declare that there is no conflict of interest that could be perceived as prejudicing the impartiality of the research reported.

\section{Funding}

This work was supported by the grant from National Natural Science Foundation of China (No. 81660246, No. 81660255, No. 81360098), the Young Scientist Training Project of Jiangxi Province, China (No. 20153BCB23032), Natural Science Foundation of Jiangxi Province (20142BAB205001) and Scientific Research Foundation of Jiujiang University (2016KJ004).

\section{References}

Brinkerhoff CR, Sharma RP \& Bourcier DR 1981 The effects of tri-otolyl phosphate (TOTP) on the immune system of mice. Ecotoxicology and Environmental Safety 5 368-376. (https://doi.org/10.1016/01476513(81)90010-5)

Britt KL, Drummond AE, Cox VA, Dyson M, Wreford NG, Jones ME, Simpson ER \& Findlay JK 2000 An age-related ovarian phenotype in mice with targeted disruption of the Cyp 19 (aromatase) gene. Endocrinology 141 2614-2623. (https://doi.org/10.1210/endo.141.7.7578)

Canipari R, O'Connell ML, Meyer G \& Strickland S 1987 Mouse ovarian granulosa cells produce urokinase-type plasminogen activator, whereas the corresponding rat cells produce tissue-type plasminogen activator. Journal of Cell Biology 105 977-981. (https://doi.org/10.1083/ jcb.105.2.977)

Chapin RE, George JD \& Lamb JC 4th 1988 Reproductive toxicity of tricresyl phosphate in a continuous breeding protocol in Swiss (CD-1) mice. Fundamental and Applied Toxicology 10 344-354. (https://doi. org/10.1016/0272-0590(88)90320-X)

Chen JX, Xu LL, Mei JH, Yu XB, Kuang HB, Liu HY, Wu YJ \& Wang JL 2012 Involvement of neuropathy target esterase in tri-ortho-cresyl phosphateinduced testicular spermatogenesis failure and growth inhibition of spermatogonial stem cells in mice. Toxicology Letters 211 54-61. (https://doi.org/10.1016/j.toxlet.2012.03.004)

Chen JX, Sun YJ, Wang P, Long DX, Li W, Li L \& Wu YJ 2013 Induction of autophagy by TOCP in differentiated human neuroblastoma cells lead to degradation of cytoskeletal components and inhibition of neurite outgrowth. Toxicology 310 92-97. (https://doi.org/10.1016/j. tox.2013.05.012)

Craig PH \& Barth ML 1999 Evaluation of the hazards of industrial exposure to tricresyl phosphate: a review and interpretation of the literature. Journal of Toxicology and Environmental Health: Part B, Critical Reviews 2 281-300. (https://doi.org/10.1080/109374099281142)

Emerick GL, Ehrich M, Jortner BS, Oliveira RV \& Deoliveira GH 2012 Biochemical, histopathological and clinical evaluation of delayed effects caused by methamidophos isoforms and TOCP in hens: ameliorative effects using control of calcium homeostasis. Toxicology 302 88-95. (https://doi.org/10.1016/j.tox.2012.08.002)

Hennet ML \& Combelles CMH 2012 The antral follicle: a microenvironment for oocyte differentiation. International Journal of Developmental Biology 56 819-831. (https://doi.org/10.1387/ijdb.120133cc)

Kabeya Y, Mizushima N, Ueno T, Yamamoto A, Kirisako T, Noda T, Kominami E, Ohsumi Y \& Yoshimoro T 2000 LC3, a mammalian homologue of yeast Apg8p, is localized in autophagosome membranes after processing. EMBO Journal 19 5720-5728. (https://doi.org/10.1093/ emboj/19.21.5720)

Kim J, Kim YC, Fang C, Russell RC, Kim JH, Fan W, Liu R, Zhong Q \& Guan KL 2013 Differential regulation of distinct Vps34 complexes by AMPK in nutrient stress and autophagy. Cell 152 290-303. (https://doi. org/10.1016/j.cell.2012.12.016)

Latendresse JR, Brooks CL \& Capen CC 1994 Pathologic effects of butylated triphenyl phosphate-based hydraulic fluid and tricresyl phosphate on the adrenal gland, ovary, and testis in the Fischer-344 rat. Toxicologic Pathology 22 341-352. (https://doi.org/10.1177/019262339402200401)

Liu ML, Wang JL, Wei J, Xu LL, Yu M, Liu XM, Ruan WL \& Chen JX 2015 Tri-ortho-cresyl phosphate- induces autophagy of rat spermatogonial stem cells. Reproduction 149 163-170. (https://doi.org/10.1530/REP14-0446)

Liu X, Xu L, Shen J, Wang J, Ruan W, Yu M \& Chen J 2016 Involvement of oxidative stress in tri-ortho-cresyl phosphate-induced autophagy of mouse Leydig TM3 cells in vitro. Reproductive Biology and Endocrinology 14 30. (https://doi.org/10.1186/s12958-016-0165-x)

Long DX \& Wu YJ 2008 Growth inhibition and induction of G (1) phase cell cycle arrest in neuroblastoma SH-SY5Y cell by tri-ortho-cresyl phosphate. Toxicology Letters 181 47-52. (https://doi.org/10.1016/j. toxlet.2008.06.871)

Mahemuti L, Chen Q, Coughlan MC, Qiao C, Chepelev NL, Florian M, Dong D, Woodworth RG, Yan J, Cao XL et al. 2018 Bisphenol A induces DSB-ATM-p53 signaling leading to cell cycle arrest, senescence, autophagy, stress response, and estrogen release in human fetal lung fibro-blasts. Archives of Toxicology 92 1453-1469. (https://doi. org/10.1007/s00204-017-2150-3) 
Matzuk MM, Finegold MJ, Su JG, Hsueh AJ \& Bradley A 1992 Alphainhibin is a tumour-suppressor gene with gonadal specificity in mice. Nature 26 313-319. (https://doi.org/10.1038/360313a0)

Quan C, Wang C, Duan P, Huang W, Chen W, Tang S \& Yang K 2017 Bisphenol A induces autophagy and apoptosis concurrently involving the Akt/mTOR pathway in testes of pubertal SD rats. Environmental Toxicology 32 1977-1989. (https://doi.org/10.1002/tox.22339)

Scarlatti F, Granata R, Meijer AJ \& Codogno P 2009 Does autophagy have a license to kill mammalian cells? Cell Death and Differentiation 16 12-20. (https://doi.org/10.1038/cdd.2008.101)

Shimizu T, Kosaka N, Murayama C, Tetsuka M \& Miyamoto A 2009 Apelin and APJ receptor expression in granulosa and theca cells during different stages of follicular development in the bovine ovary: involvement of apoptosis and hormonal regulation. Animal Reproduction Science $\mathbf{1 1 6}$ 28-37. (https://doi.org/10.1016/j.anireprosci.2009.01.009)

Smith MI, Elvove E, Valaer PJ, Frazier WH \& Mallory GE 1930 Pharmacologic and chemical studies of the cause of the so-called ginger paralysis: a preliminary report. Public Health Reports 45 1703-1716. (https://doi.org/10.2307/4579730)

Somkuti SG, Lapadula DM, Chapin RE, Lamb JC 4th \& Abou-Donia MB $1987 a$ Reproductive tract lesions resulting from subchronic administration (63 days) of tri-o-cresyl phosphate in male rats. Toxicology and Applied Pharmacology 89 49-63. (https://doi.org/10.1016/0041008X(87)90175-X)

Somkuti SG, Lapadula DM, Chapin RE, Lamb JC 4th \& Abou-Donia MB $1987 b$ Testicular toxicity following oral administration of tri-o-cresyl phosphate (TOCP) in roosters. Toxicology Letters 37 279-290. (https:// doi.org/10.1016/0378-4274(87)90143-3)

Somkuti SG, Lapadula DM, Chapin RE \& Abou-Donia MB 1991 Light and electron microscopic evidence of tri-o-cresyl phosphate (TOCP)mediated testicular toxicity in Fischer 344 rats. Toxicology and Applied Pharmacology 107 35-46. (https://doi.org/10.1016/0041008X(91)90328-C)

Sun YY, Shen JC, Zeng L, Yang D, Shao SX, Wang JL, Wei J, Xiong JP \& Chen JX 2018 Role of autophagy in di-2-ethylhexyl phthalate (DEHP)- induced apoptosis in mouse Leydig cells. Environmental Pollution 243 563-572. (https://doi.org/10.1016/j.envpol.2018.08.089)

Winder C \& Balouet JC 2002 The toxicity of commercial jet oils. Environmental Research 89 146-164. (https://doi.org/10.1006/ enrs.2002.4346)

Wu YJ \& Leng XF 2000 Effects of tri-o-cresyl phosphate on serum estrogen and progesterone concentration and ATPase activity in the shell gland of adult hens. Chemosphere 41 183-186. (https://doi.org/10.1016/S00456535(99)00409-9)

Xu LL, Long CY, Wang JL, Yu M \& Chen JX 2016 Involvement of oxidative stress in tri-ortho-cresyl phosphate-induced liver injury in male mice. Human and Experimental Toxicology 35 1093-1101. (https://doi. org/10.1177/0960327115621363)

Yang MY \& Fortune JE 2007 Vascular endothelial growth factor stimulates the primary to secondary follicle transition in bovine follicles in vitro. Molecular Reproduction and Development 74 1095-1104. (https://doi. org/10.1002/mrd.20633)

Yao X, Sha S, Wang Y, Sun X, Cao J, Kang J, Jiang L, Chen M \& Ma Y 2016 Perfluorooctane sulfonate induces autophagy-dependent apoptosis through spinster 1-mediated lysosomal-mitochondrial axis and impaired mitophagy. Toxicological Sciences 153 198-211. (https://doi. org/10.1093/toxsci/kfw118)

Zhang LP, Wang QS, Guo X, Zhu YJ, Zhou GZ \& Xie KQ 2007 Timedependent changes of lipid peroxidation and antioxidative status in nerve tissues of hens treated with tri-ortho-cresyl phosphate (TOCP). Toxicology 239 45-52. (https://doi.org/10.1016/j.tox.2007.06.091)

Received 28 August 2018

First decision 19 October 2018

Revised manuscript received 10 April 2019

Accepted 23 April 2019 